\title{
MATHEMATICAL ASPECTS OF MEAN FIELD SPIN GLASS THEORY
}

\author{
Francesco Guerra* \\ Dipartimento di Fisica, Università di Roma "La Sapienza" \\ and INFN, Sezione di Roma1, Piazzale A. Moro 2, 00185 Roma, Italy
}

November 5, 2018

\begin{abstract}
A comprehensive review will be given about the rich mathematical structure of mean field spin glass theory, mostly developed, until now, in the frame of the methods of theoretical physics, based on deep physical intuition and hints coming from numerical simulation. Central to our treatment is a very simple and yet powerful interpolation method, allowing to compare different probabilistic schemes, by using convexity and positivity arguments. In this way we can prove the existence of the thermodynamic limit for the free energy density of the system, a long standing open problem. Moreover, in the frame of a generalized variational principle, we can show the emergency of the Derrida-Ruelle random probability cascades, leading to the form of free energy given by the celebrated Parisi Ansatz. All these results seem to be in full agreement with the mechanism of spontaneous replica symmetry breaking as developed by Giorgio Parisi.
\end{abstract}

* e-mail: francesco.guerra@roma1.infn.it 


\section{Introduction}

The mean field model for spin glasses, introduced by David Sherrington and Scott Kirkpatrick more that thirty years ago [1], 2], is a celebrated model. Hundreds and hundreds of articles have been devoted to its study during the years, appearing in the theoretical physics literature.

The relevance of the model stems surely from the fact that it is intended to represent some important features of the physical spin glass systems, of great interest for their peculiar properties, exhibiting a new magnetic phase, where magnetic moments are frozen into disordered equilibrium orientations, without any long-range order. See for example [3] for a very readable review about the physical properties of spin glasses.

But another important source of interest is connected with the fact that disordered systems, of the Sherrington-Kirkpatrick type, and their generalizations, seems to play a very important role for theoretical and practical assessments about hard optimization problems, as it is shown for example by Mark Mézard, Giorgio Parisi and Riccardo Zecchina in [4.

It is interesting to remark that the original paper was entitled "Solvable Model of a Spin-Glass", while a previous draft, as I have it from David Sherrington, contained even the stronger denomination "Exactly Solvable". However, it turned out that the very natural solution devised by the authors was valid only at high temperatures, or large external magnetic fields. While, at low temperatures, the proposed solution exhibited a nonphysical drawback

given by a negative entropy, as properly recognized by the authors in their very first paper.

It took some years to find an acceptable solution. This was done by Giorgio Parisi in a series of papers, by marking a radical departure from the previous methods. In fact, a very deep method of "spontaneous replica symmetry breaking" was developed. As a consequence the physical content of the theory was encoded in a functional order parameter of new type, and a remarkable structure emerged for the pure states of the theory, a kind of hierarchical, ultrametric organization. These very interesting developments, due to Giorgio Parisi, and his coworkers, are explained in a lucid way in the classical book [5]. Part of this structure will be recalled in the following.

It is important to remark that Parisi solution is presented in the form of an ingenious and clever Ansatz. Until few years ago it was not known whether this Ansatz would give the true solution for the model, in the so called thermodynamic limit, when the size of the system becomes infinite, or it would be only a very good approximation for the true solution.

The general structure offered by the Parisi solution, and their possible generalizations for similar models, exhibit an extremely rich and interesting 
mathematical content. Very appropriately, Michel Talagrand has inserted a strongly suggestive sentence in the title to his recent book [6]:"Spin glasses: a challenge for mathematicians".

As a matter of fact, how to face this challenge is a very difficult problem. Here we would like to recall the main features of a very powerful method, yet extremely simple in its very essence, based on a comparison and interpolation argument on sets of Gaussian random variables.

The method found its first simple application in [7], where it was shown that the Sherrington-Kirkpatrick replica symmetric approximate solution was a rigourous lower bound for the quenched free energy of the system, uniformly in the size. Then, it was possible to reach a long waited result [8]: the convergence of the free energy density in the thermodynamic limit, by an intermediate step where the quenched free energy was shown to be subadditive in the size of the system.

Moreover, still by interpolation on families of Gaussian random variables, the first mentioned result was extended to give a rigorous proof that the expression given by the Parisi Ansatz is also a lower bound for the quenched free energy of the system, uniformly in the size [9]. The method gives not only the bound, but also the explicit form of the correction in a quite involved form. As a recent and very important result, along the task of facing the challenge, Michel Talagrand has been able to dominate these correction terms, showing that they vanish in the thermodynamic limit. This milestone achievement was firstly announced in a short note [10], containing only a synthetic sketch of the proof, and then presented with all details in a long paper to be published on Annals of Mathematics [11].

The interpolation method is also at the basis of the far reaching generalized variational principle proven by Michel Aizenman, Robert Sims and Shannon Starr in 12 .

In our presentation, we will try to be as self-contained as possible. We will give all definitions, explain the basic structure of the interpolation method, and show how some of the results are obtained. We will concentrate mostly on questions connected with the free energy, its properties of subadditivity, the existence of the infinite volume limit, and the replica bounds.

For the sake of comparison, and in order to provide a kind of warm up, we will recall also some features of the standard elementary mean field model of ferromagnetism, the so called Curie-Weiss model. We will concentrate also here on the free energy, and systematically exploit elementary comparison and interpolation arguments. This will show the strict analogy between the treatment of the ferromagnetic model and the developments in the mean field spin glass case. Basic roles will be played in the two cases, but with different expressions, by positivity and convexity properties. 
The organization of the paper is as follows. In Section 2, we introduce the ferromagnetic model and discuss behavior and properties of the free energy in the thermodynamic limit, by emphasing, in this very elementary case, the comparison and interpolation methods that will be also exploited, in a different context, in the spin glass case.

Section 3 is devoted to the basic features of the mean field spin glass models, by introducing all necessary definitions.

In Section 4, we introduce the Gaussian comparison and interpolation method, by giving simple applications to the existence of the infinite volume limit of the quenched free energy [8], and to the proof of general variational bounds, by following the useful strategy developed in [12].

Section 5 will briefly recall the main features of the Parisi representation, and will state the main theorem concerning the free energy.

Finally, Section 6 will be devoted to conclusions and outlook for future developments.

It is a pleasure to thank the Organizing Committee, and in particular Professor Ari Laptev, for the kind invitation to talk in a such stimulating cultural athmosphere.

\section{The mean field ferromagnetic model. Struc- ture and results.}

The mean field ferromagnetic model is among the simplest models of statistical mechanics. However, it contains very interesting features, in particular a phase transition, characterized by spontaneous magnetization, at low temperatures. We refer to standard textbooks, for example [13], for a full treatment, and a complete appreciation of the model in the frame of the theory of ferromagnetism. Here we consider only some properties of the free energy, easily obtained through comparison methods.

The generic configuration of the mean field ferromagnetic model is defined through Ising spin variables $\sigma_{i}= \pm 1$, attached to each site $i=1,2, \ldots, N$.

The Hamiltonian of the model, in some external field of strength $h$, is given by the mean field expression

$$
H_{N}(\sigma, h)=-\frac{1}{N} \sum_{(i, j)} \sigma_{i} \sigma_{j}-h \sum_{i} \sigma_{i} .
$$

Here, the first sum extends to all $N(N-1) / 2$ site couples, an the second to all sites. 
For a given inverse temperature $\beta$, let us now introduce the partition function $Z_{N}(\beta, h)$ and the free energy per site $f_{N}(\beta, h)$, according to the well known definitions

$$
\begin{aligned}
& Z_{N}(\beta, h)=\sum_{\sigma_{1} \ldots \sigma_{N}} \exp \left(-\beta H_{N}(\sigma, h)\right), \\
& -\beta f_{N}(\beta, h)=N^{-1} E \log Z_{N}(\beta, h) .
\end{aligned}
$$

It is also convenient to define the average spin magnetization

$$
m=\frac{1}{N} \sum_{i} \sigma_{i}
$$

Then, it is immediately seen that the Hamiltonian in (11) can be equivalently written as

$$
H_{N}(\sigma, h)=-\frac{1}{2} N m^{2}-h \sum_{i} \sigma_{i},
$$

where an unessential constant term has been neglected. In fact we have

$$
\sum_{(i, j)} \sigma_{i} \sigma_{j}=\frac{1}{2} \sum_{i, j ; i \neq j} \sigma_{i} \sigma_{j}=\frac{1}{2} N^{2} m^{2}-\frac{1}{2} N,
$$

where the sum over all couples has been equivalently written as one half the sum over all $i, j$ with $i \neq j$, and the diagonal terms with $i=j$ have been added and subtracted out. Notice that they give a constant because $\sigma_{i}^{2}=1$.

Therefore, the partition function in (2) can be equivalently substituted by the expression

$$
Z_{N}(\beta, h)=\sum_{\sigma_{1} \ldots \sigma_{N}} \exp \left(-\frac{1}{2} \beta N m^{2}\right) \exp \left(\beta h \sum_{i} \sigma_{i}\right),
$$

which will be our starting point.

Our interest will be in the $\lim _{N \rightarrow \infty} N^{-1} \log Z_{N}(\beta, h)$. To this purpose, let us establish the important subadditivity property, holding for the splitting of the big $\mathrm{N}$ site system in two smaller $N_{1}$ site and $N_{2}$ site systems, respectively, with $N=N_{1}+N_{2}$,

$$
\log Z_{N}(\beta, h) \leq \log Z_{N_{1}}(\beta, h)+\log Z_{N_{2}}(\beta, h) .
$$

The proof is very simple. Let us denote, in the most natural way, by $\sigma_{1}, \ldots, \sigma_{N_{1}}$ the spin variables for the first subsystem, and by $\sigma_{N_{1}+1}, \ldots, \sigma_{N}$ 
the $N_{2}$ spin variables of the second subsystem. Introduce also the subsystem magnetizations $m_{1}$ and $m_{2}$, by adapting the definition (4) to the smaller systems, in such a way that

$$
N m=N_{1} m_{1}+N_{2} m_{2}
$$

Therefore, we see that the large system magnetization $m$ is the linear convex combination of the smaller system ones, according to the obvious

$$
m=\frac{N_{1}}{N} m_{1}+\frac{N_{2}}{N} m_{2} .
$$

Since the mapping $m \rightarrow m^{2}$ is convex, we have also the general bound, holding for all values of the $\sigma$ variables

$$
m^{2} \leq \frac{N_{1}}{N} m_{1}^{2}+\frac{N_{2}}{N} m_{2}^{2}
$$

Then, it is enough to substitute the inequality in the definition (17) of $Z_{N}(\beta, h)$, and recognize that we achieve factorization with respect to the two subsystems, and therefore the inequality $Z_{N} \leq Z_{N_{1}} Z_{N_{2}}$. So we have established (8)). From subadditivity, the existence of the limit follows by a simple argument, as explained for example in [14]. In fact, we have

$$
\lim _{N \rightarrow \infty} N^{-1} \log Z_{N}(\beta, h)=\inf _{N} N^{-1} \log Z_{N}(\beta, h) .
$$

Now we will calculate explicitely this limit, by introducing an order parameter $M$, a trial function, and an appropriate variational scheme. In order to get a lower bound, we start from the elementary inequality $m^{2} \geq 2 m M-$ $M^{2}$, holding for any value of $m$ and $M$. By inserting the inequality in the definition (7) we arrive at a factorization of the sum over $\sigma$ 's. The sum can be explicitely calculated, and we arrive immediately to the lower bound, uniform in the size of the system,

$$
N^{-1} \log Z_{N}(\beta, h) \geq \log 2+\log \cosh \beta(h+M)-\frac{1}{2} \beta M^{2},
$$

holding for any value of the trial order parameter $M$. Clearly it is convenient to take the supremum over $M$. Then we establish the optimal uniform lower bound

$$
N^{-1} \log Z_{N}(\beta, h) \geq \sup _{M}\left(\log 2+\log \cosh \beta(h+M)-\frac{1}{2} \beta M^{2}\right) .
$$

It is simple to realize that the supremum coincides with the limit as $N \rightarrow \infty$. To this purpose we follow the following simple procedure. Let 
us consider all possible values of the variable $m$. There are $N+1$ of them, corresponding to any number $K$ of possible spin flips, starting from a given $\sigma$ configuration, $K=0,1, \ldots, N$. Let us consider the trivial decomposition of the identity, holding for any $m$,

$$
1=\sum_{M} \delta_{m M}
$$

where $M$ in the sum runs over the $N+1$ possible values of $m$, and $\delta$ is Kroneker delta, beeing equal to 1 if $M=N$, and zero otherwise. Let us now insert (15) in the definition (17) of the partition function inside the sum over $\sigma$ 's, and invert the two sums. Because of the forcing $m=M$ given by the $\delta$, we can write $m^{2}=2 m M-M^{2}$ inside the sum. Then if we neglect the $\delta$, by using the trivial $\delta \leq 1$, we have un upper bound, where the sum over $\sigma$ 's can be explicitily performed as before. Then it is enough to take the upper bound with respect to $M$, and consider that there are $N+1$ terms in the now trivial sum over $M$, in order to arrive at the upper bound

$N^{-1} \log Z_{N}(\beta, h) \leq \sup _{M}\left(\log 2+\log \cosh \beta(h+M)-\frac{1}{2} \beta M^{2}\right)+N^{-1} \log (N+1)$.

Therefore, by going to the limit as $N \rightarrow \infty$, we can collect all our results in the form of the following theorem giving the full characterization of the thermodynamic limit of the free energy.

Theorem 1. For the mean field ferromagnetic model we have

$$
\begin{aligned}
& \lim _{N \rightarrow \infty} N^{-1} \log Z_{N}(\beta, h)=\inf _{N} N^{-1} \log Z_{N}(\beta, h) \\
& =\sup _{M}\left(\log 2+\log \cosh \beta(h+M)-\frac{1}{2} \beta M^{2}\right) .
\end{aligned}
$$

This ends our discussion about the free energy in the ferromagnetic model. Now we are ready to attack the much more difficult spin glass model. But it will be surprising to see that, by following a simple extension of the methods here described, we will arrive to similar results.

\section{The basic definitions for the mean field spin glass model}

As in the ferromagnetic case, the generic configuration of the mean field spin glass model is defined through Ising spin variables $\sigma_{i}= \pm 1$, attached to each site $i=1,2, \ldots, N$. 
But now there is an external quenched disorder given by the $N(N-1) / 2$ independent and identical distributed random variables $J_{i j}$, defined for each couple of sites. For the sake of simplicity, we assume each $J_{i j}$ to be a centered unit Gaussian with averages $E\left(J_{i j}\right)=0, E\left(J_{i j}^{2}\right)=1$. By quenched disorder we mean that the $J$ have a kind of stochastic external influence on the system, without partecipating to the thermal equilibrium.

Now the Hamiltonian of the model, in some external field of strength $h$, is given by the mean field expression

$$
H_{N}(\sigma, h, J)=-\frac{1}{\sqrt{N}} \sum_{(i, j)} J_{i j} \sigma_{i} \sigma_{j}-h \sum_{i} \sigma_{i} .
$$

Here, the first sum extends to all site couples, an the second to all sites. Notice the $\sqrt{N}$ necessary to ensure a good thermodynamic behavior to the free energy.

For a given inverse temperature $\beta$, let us now introduce the disorder dependent partition function $Z_{N}(\beta, h, J)$ and the quenched average of the free energy per site $f_{N}(\beta, h)$, according to the definitions

$$
\begin{aligned}
& Z_{N}(\beta, h, J)=\sum_{\sigma_{1} \ldots \sigma_{N}} \exp \left(-\beta H_{N}(\sigma, h, J)\right), \\
& -\beta f_{N}(\beta, h)=N^{-1} E \log Z_{N}(\beta, h, J) .
\end{aligned}
$$

Notice that in (21) the average $E$ with respect to the external noise is made after the $\log$ is taken. This procedure is called quenched averaging. It represents the physical idea that the external noise does not partecipate to the thermal equilibrium. Only the $\sigma$ 's are thermalized.

For the sake of simplicity, it is also convenient to write the partition function in the following equivalent form. First of all let us introduce a family of centered Gaussian random variables $\kappa(\sigma)$, indexed by the configurations $\sigma$, and characterized by the covariances

$$
E\left(\kappa(\sigma) \kappa\left(\sigma^{\prime}\right)\right)=q^{2}\left(\sigma, \sigma^{\prime}\right)
$$

where $q\left(\sigma, \sigma^{\prime}\right)$ are the overlaps between two generic configurations, defined by

$$
q\left(\sigma, \sigma^{\prime}\right)=N^{-1} \sum_{i} \sigma_{i} \sigma_{i}^{\prime}
$$

with the obvious bounds $-1 \leq q\left(\sigma, \sigma^{\prime}\right) \leq 1$, and the normalization $q(\sigma, \sigma)=$ 1. Then, starting from the definition (19), it is immediately seen that the partition function in (20) can be also written, by neglecting unessential constant 
terms, in the form

$$
Z_{N}(\beta, h, J)=\sum_{\sigma_{1} \ldots \sigma_{N}} \exp \left(\beta \sqrt{\frac{N}{2}} \kappa(\sigma)\right) \exp \left(\beta h \sum_{i} \sigma_{i}\right)
$$

which will be the starting point of our treatment.

\section{Gaussian comparison and applications}

Our basic comparison argument will be based on the following very simple theorem.

Theorem 2. Let $U_{i}$ and $\hat{U}_{i}$, for $i=1, \ldots, K$, be independent families of centered Gaussian random variables, whose covariances satisfy the inequalities for generic configurations

$$
E\left(U_{i} U_{j}\right) \equiv S_{i j} \geq E\left(\hat{U}_{i} \hat{U}_{j}\right) \equiv \hat{S}_{i j}
$$

and the equalities along the diagonal

$$
E\left(U_{i} U_{i}\right) \equiv S_{i i}=E\left(\hat{U}_{i} \hat{U}_{i}\right) \equiv \hat{S}_{i i}
$$

then for the quenched averages we have the inequality in the opposite sense

$$
E \log \sum_{i} w_{i} \exp \left(U_{i}\right) \leq E \log \sum_{i} w_{i} \exp \left(\hat{U}_{i}\right)
$$

where the $w_{i} \geq 0$ are the same in the two expressions.

The proof is extremely simple and amounts to a straigthforward calculation. In fact, let us consider the interpolating expression

$$
E \log \sum_{i} w_{i} \exp \left(\sqrt{t} U_{i}+\sqrt{1-t} \hat{U}_{i}\right)
$$

where $0 \leq t \leq 1$. Clearly the two expressions under comparison correspond to the values $t=0$ and $t=1$ respectively. By taking the derivative with respect to $t$, and then integrating by parts with respect to the Gaussian variables, we immediately see that the interpolating function is nonincreasing in $t$, and the theorem follows. On the other hand, considerations of this kind are present in the mathematical literature of some years ago. Two typical references are [15] and [16].

We give here some striking applications of the basic comparison Theorem. In [8] we have given a very simple proof of a long waited result, about the 
convergence of the free energy per site in the thermodynamic limit. Let us show the argument. Let us consider a system of size $N$ and two smaller systems of sizes $N_{1}$ and $N_{2}$ respectively, with $N=N_{1}+N_{2}$, as before in the ferromagnetic case. Let us now compare

$$
E \log Z_{N}(\beta, h, J)=E \log \sum_{\sigma_{1} \ldots \sigma_{N}} \exp \left(\beta \sqrt{\frac{N}{2}} \kappa(\sigma)\right) \exp \left(\beta h \sum_{i} \sigma_{i}\right),
$$

with

$$
\begin{aligned}
& E \log \sum_{\sigma_{1} \ldots \sigma_{N}} \exp \left(\beta \sqrt { \frac { N _ { 1 } } { 2 } } \kappa ^ { ( 1 ) } ( \sigma ^ { ( 1 ) } ) \operatorname { e x p } \left(\beta \sqrt{\frac{N_{2}}{2}} \kappa^{(2)}\left(\sigma^{(2)}\right) \exp \left(\beta h \sum_{i} \sigma_{i}\right)\right.\right. \\
& \equiv E \log Z_{N_{1}}(\beta, h, J)+E \log Z_{N_{2}}(\beta, h, J)
\end{aligned}
$$

where $\sigma^{(1)}$ are the $\left(\sigma_{i}, i=1, \ldots, N_{1}\right)$, and $\sigma^{(2)}$ are the $\left(\sigma_{i}, i=N_{1}+1, \ldots, N\right)$. Covariances for $\kappa^{(1)}$ and $\kappa^{(2)}$ are expressed as in (22) , but now the overlaps are substituted with the partial overlaps of the first and second block, $q_{1}$ and $q_{2}$ respectively. It is very simple to apply the comparison theorem. All one has to do is to observe that the obvious

$$
N q=N_{1} q_{1}+N_{2} q_{2}
$$

analogous to (9), implies, as in (11),

$$
q^{2} \leq \frac{N_{1}}{N} q_{1}^{2}+\frac{N_{2}}{N} q_{2}^{2}
$$

Therefore, the comparison gives the superaddivity property, to be compared with (8),

$$
E \log Z_{N}(\beta, h, J) \geq E \log Z_{N_{1}}(\beta, h, J)+E \log Z_{N_{2}}(\beta, h, J) .
$$

From the superaddivity property the existence of the limit follows in the form

$$
\lim _{N \rightarrow \infty} N^{-1} E \log Z_{N}(\beta, h, J)=\sup _{N} N^{-1} E \log Z_{N}(\beta, h, J),
$$

to be compared with (12).

The second application is in the form of the Aizenman-Sims-Starr generalized variational principle. Here, we will need to introduce some auxiliary system. The denumerable configuration space is given by the values of $\alpha=1,2, \ldots$. We introduce also a probability measure $w_{\alpha}$ for the $\alpha$ system, and suitably defined overlaps between two generic configurations $p\left(\alpha, \alpha^{\prime}\right)$, 
with $p(\alpha, \alpha)=1$. A family of centered Gaussian random variables $\hat{\kappa}(\alpha)$, now indexed by the configurations $\alpha$, will be defined by the covariances

$$
E\left(\hat{\kappa}(\alpha) \hat{\kappa}\left(\alpha^{\prime}\right)\right)=p^{2}\left(\alpha, \alpha^{\prime}\right) .
$$

We will need also a family of centered Gaussian random variables $\eta_{i}(\alpha)$, indexed by the sites $i$ of our original system and the configurations $\alpha$ of the auxiliary system, so that

$$
E\left(\eta_{i}(\alpha) \eta_{i^{\prime}}\left(\alpha^{\prime}\right)\right)=\delta_{i i^{\prime}} p\left(\alpha, \alpha^{\prime}\right)
$$

Both the probability measure $w_{\alpha}$, and the overlaps $p\left(\alpha, \alpha^{\prime}\right)$ could depend on some additional external quenched noise, that does not appear explicitely in our notation.

In the following, we will denote by $E$ averages with respect to all random variables involved.

In order to start the comparison argument, we will consider firstly the case where the two $\sigma$ and $\alpha$ systems are not coupled, so to appear factorized in the form

$$
\begin{aligned}
& E \log \sum_{\sigma_{1} \ldots \sigma_{N}} \sum_{\alpha} w_{\alpha} \exp \left(\beta \sqrt{\frac{N}{2}} \kappa(\sigma)\right) \exp \left(\beta \sqrt{\frac{N}{2}} \hat{\kappa}(\alpha)\right) \exp \left(\beta h \sum_{i} \sigma_{i}\right) \\
& \equiv E \log Z_{N}(\beta, h, J)+E \log \sum_{\alpha} w_{\alpha} \exp \left(\beta \sqrt{\frac{N}{2}} \hat{\kappa}(\alpha)\right) .
\end{aligned}
$$

In the second case the $\kappa$ fields are suppressed and the coupling between the two systems will be taken in a very simple form, by allowing the $\eta$ field to act as an external field on the $\sigma$ system. In this way the $\sigma$ 's appear as factorized, and the sums can be explicitely performed. The chosen form for the second term in the comparison is

$$
\begin{aligned}
& E \log \sum_{\sigma_{1} \ldots \sigma_{N}} \sum_{\alpha} w_{\alpha} \exp \left(\beta \sum_{i} \eta_{i}(\alpha) \sigma_{i}\right) \exp \left(\beta h \sum_{i} \sigma_{i}\right) \\
& \equiv N \log 2+E \log \sum_{\alpha} w_{\alpha}\left(c_{1} c_{2} \ldots c_{N}\right),
\end{aligned}
$$

where we have defined

$$
c_{i}=\cosh \beta\left(h+\eta_{i}(\alpha)\right)
$$

as arising from the sums over $\sigma^{\prime}$ 's. 
Now we apply the comparison Theorem. In the first case, the covariances involve the sums of squares of overlaps

$$
\frac{1}{2}\left(q^{2}\left(\sigma, \sigma^{\prime}\right)+p^{2}\left(\alpha, \alpha^{\prime}\right)\right) .
$$

In the second case, a very simple calculation shows that the covariances involve the overlap products

$$
q\left(\sigma, \sigma^{\prime}\right) p\left(\alpha, \alpha^{\prime}\right)
$$

Therefore, the comparison is very easy and, by collecting all expressions, we end up with the useful estimate, as in [12, holding for any auxiliary system as defined before,

$$
\begin{aligned}
& N^{-1} E \log Z_{N}(\beta, h, J) \leq \\
& \log 2+N^{-1}\left(E \log \sum_{\alpha} w_{\alpha}\left(c_{1} c_{2} \ldots c_{N}\right)-E \log \sum_{\alpha} w_{\alpha} \exp \left(\beta \sqrt{\frac{N}{2}} \hat{\kappa}(\alpha)\right)\right) .
\end{aligned}
$$

\section{The Parisi representation for the free en- ergy}

We refer to the original paper [17], and to the extensive review given in [5], for the general motivations, and the derivation of the broken replica Ansatz, in the frame of the ingenious replica trick. Here we limit ourselves to a synthetic description of its general structure, independently from the replica trick

First of all, let us introduce the convex space $\mathcal{X}$ of the functional order parameters $x$, as nondecreasing functions of the auxiliary variable $q$, both $x$ and $q$ taking values on the interval $[0,1]$, i.e.

$$
\mathcal{X} \ni x:[0,1] \ni q \rightarrow x(q) \in[0,1] .
$$

Notice that we call $x$ the function, and $x(q)$ its values. We introduce a metric on $\mathcal{X}$ through the $L^{1}([0,1], d q)$ norm, where $d q$ is the Lebesgue measure.

For our purposes, we will consider the case of piecewise constant functional order parameters, characterized by an integer $K$, and two sequences $q_{0}, q_{1}, \ldots, q_{K}, m_{1}, m_{2}, \ldots, m_{K}$ of numbers satisfying

$$
0=q_{0} \leq q_{1} \leq \ldots \leq q_{K-1} \leq q_{K}=1,0 \leq m_{1} \leq m_{2} \leq \ldots \leq m_{K} \leq 1
$$


such that

$$
\begin{aligned}
x(q)=m_{1} \text { for } 0=q_{0} \leq q<q_{1}, x(q)=m_{2} & \text { for } \quad q_{1} \leq q<q_{2}, \\
\ldots, x(q)=m_{K} & \text { for } \quad q_{K-1} \leq q \leq q_{K} .
\end{aligned}
$$

In the following, we will find convenient to define also $m_{0} \equiv 0$, and $m_{K+1} \equiv 1$. The replica symmetric case of Sherrington and Kirkpatrick corresponds to

$$
K=2, q_{1}=\bar{q}, m_{1}=0, m_{2}=1 .
$$

Let us now introduce the function $f$, with values $f(q, y ; x, \beta)$, of the variables $q \in[0,1], y \in R$, depending also on the functional order parameter $x$, and on the inverse temperature $\beta$, defined as the solution of the nonlinear antiparabolic equation

$$
\left(\partial_{q} f\right)(q, y)+\frac{1}{2}\left(\partial_{y}^{2} f\right)(q, y)+\frac{1}{2} x(q)\left(\partial_{y} f\right)^{2}(q, y)=0
$$

with final condition

$$
f(1, y)=\log \cosh (\beta y) .
$$

Here, we have stressed only the dependence of $f$ on $q$ and $y$.

It is very simple to integrate Eq. (47) when $x$ is piecewise constant. In fact, consider $x(q)=m_{a}$, for $q_{a-1} \leq q \leq q_{a}$, firstly with $m_{a}>0$. Then, it is immediately seen that the correct solution of Eq. (47) in this interval, with the right final boundary condition at $q=q_{a}$, is given by

$$
f(q, y)=\frac{1}{m_{a}} \log \int \exp \left(m_{a} f\left(q_{a}, y+z \sqrt{q_{a}-q}\right)\right) d \mu(z),
$$

where $d \mu(z)$ is the centered unit Gaussian measure on the real line. On the other hand, if $m_{a}=0$, then (47) loses the nonlinear part and the solution is given by

$$
f(q, y)=\int f\left(q_{a}, y+z \sqrt{q_{a}-q}\right) d \mu(z),
$$

which can be seen also as deriving from (49) in the limit $m_{a} \rightarrow 0$. Starting from the last interval $K$, and using (49) iteratively on each interval, we easily get the solution of (47), (48), in the case of piecewise order parameter $x$, as in (45).

Now we introduce the following important definitions. The trial auxiliary function, associated to a given mean field spin glass system, as described in Section 3, depending on the functional order parameter $x$, is defined as

$$
\log 2+f(0, h ; x, \beta)-\frac{\beta^{2}}{2} \int_{0}^{1} q x(q) d q .
$$


Notice that in this expression the function $f$ appears evaluated at $q=0$, and $y=h$, where $h$ is the value of the external magnetic field. This trial expression shoul be considered as the analog of that appearing in (13) for the ferromagnetic case.

The Parisi spontaneously broken replica symmetry expression for the free energy is given by the definition

$$
-\beta f_{P}(\beta, h) \equiv \inf _{x}\left(\log 2+f(0, h ; x, \beta)-\frac{\beta^{2}}{2} \int_{0}^{1} q x(q) d q\right),
$$

where the infimum is taken with respect to all functional order parameters $x$. Notice that the infimum appears here, as compared to the supremum in the ferromagnetic case.

In [9], by exploiting a kind of generalized comparison argument, involving a suitably defined interpolation function, we have established the following important result.

Theorem 3. For all values of the inverse temperature $\beta$, and the external magnetic field $h$, and for any functional order parameter $x$, the following bound holds

$$
N^{-1} E \log Z_{N}(\beta, h, J) \leq \log 2+f(0, h ; x, \beta)-\frac{\beta^{2}}{2} \int_{0}^{1} q x(q) d q,
$$

uniformly in $N$. Consequently, we have also

$$
N^{-1} E \log Z_{N}(\beta, h, J) \leq \inf _{x}\left(\log 2+f(0, h ; x, \beta)-\frac{\beta^{2}}{2} \int_{0}^{1} q x(q) d q\right),
$$

uniformly in $N$.

However, this result can be understood also in the frame of the generalized variational principle established by Aizenman-Sims-Starr and described before.

In fact, one can easily show that there exist an $\alpha$ systems such that

$$
\begin{gathered}
N^{-1} E \log \sum_{\alpha} w_{\alpha}\left(c_{1} c_{2} \ldots c_{N}\right) \equiv f(0, h ; x, \beta), \\
N^{-1} E \log \sum_{\alpha} w_{\alpha} \exp \left(\beta \sqrt{\frac{N}{2}} \hat{\kappa}(\alpha)\right) \equiv \frac{\beta^{2}}{2} \int_{0}^{1} q x(q) d q,
\end{gathered}
$$

uniformly in $N$. This result stems from previous work of Derrida, Ruelle, Neveu, Bolthausen, Sznitman, Aizenman, Talagrand, Bovier, and others, 
and in a sense is implicit in the treatment given in [5]. We plan to deal with this important representation in a forthcoming note.

We see that the estimate in Theorem 3 are also a consequence of the generalized variational principle.

Up to this point we have seen how to obtain upper bounds. The problem arises whether, as in the ferromagnetic case, we can also get lower bounds, so to shrink the thermodynamic limit to the value given by the $\inf _{x}$ in Theorem [3. After a short announcement in [10, Michel Talagrand wrote an extended paper [11, to appear on Annals of Mathematics, where the complete proof of the control of the lower bound is firmly established. We refer to the original paper for the complete details of this remarkable achievement. About the methods, here we only recall that in [9] we have given also the corrections to the bounds appearing in Theorem 3, albeit in a quite complicated form. Talagrand, with great courage, has been able to establish that these corrections do in fact vanish in the thermodynamic limit.

In conclusion, we can establish the following extension of Theorem 1 to spin glasses.

Theorem 4. For the mean field spin glass model we have

$$
\begin{aligned}
& \lim _{N \rightarrow \infty} N^{-1} E \log Z_{N}(\beta, h, J)=\sup _{N} N^{-1} E \log Z_{N}(\beta, h, J) \\
& =\inf _{x}\left(\log 2+f(0, h ; x, \beta)-\frac{\beta^{2}}{2} \int_{0}^{1} q x(q) d q\right) .
\end{aligned}
$$

\section{Conclusion and outlook for future develop- ments}

As we have seen, in these last few years there has been an impressive progress in the understanding of the mathematical structure of spin glass models, mainly due to the systematic exploration of comparison and interpolation methods. However many important problems are still open. The most important one is to establish rigorously the full hierarchical ultrametric organization of the overlap distributions, as appears in Parisi theory, and to fully understand the decomposition in pure states of the glassy phase, at low temperatures.

Moreover, is would be important to extend these methods to other important disordered models as for example neural networks. Here the difficulty is that the positivity arguments, so essential in comparison methods, do not seem to emerge naturally inside the structure of the theory.

We plan to report on these problems in future works. 


\section{Acknowledgments}

We gratefully acknowledge useful conversations with Michael Aizenman, Pierluigi Contucci, Giorgio Parisi and Michel Talagrand. The strategy explained in this paper grew out from a systematic exploration of comparison and interpolation methods, developed in collaboration with Fabio Lucio Toninelli.

This work was supported in part by MIUR (Italian Minister of Instruction, University and Research), and by INFN (Italian National Institute for Nuclear Physics).

\section{References}

[1] D. Sherrington and S. Kirkpatrick, Solvable Model of a Spin-Glass Phys. Rev. Lett. 35, 1792-1796 (1975).

[2] S. Kirkpatrick and D. Sherrington, Infinite-ranged models of spin-glasses, Phys. Rev. B17, 4384-4403 (1978).

[3] D.L. Stein, Disordered Systems: Mostly Spin Glasses, in: Lectures in the Sciences of Complexity, ed. D.L. Stein, Addison-Wesley, NY, 1989.

[4] M. Mézard, G. Parisi and R. Zecchina, Analytic and Algorithmic Solution of Random Satisfiability Problems, Science 297, 812 (2002).

[5] M. Mézard, G. Parisi and M. A. Virasoro, Spin glass theory and beyond, World Scientific, Singapore, 1987.

[6] M. Talagrand, Spin glasses: a challenge for mathematicians. Mean field models and cavity method, Springer-Verlag, Berlin (2003).

[7] F. Guerra, Sum rules for the free energy in the mean field spin glass model, Fields Institute Communications 30, 161 (2001).

[8] F. Guerra and F. L. Toninelli, The Thermodynamic Limit in Mean Field Spin Glass Models, Commun. Math. Phys. 230, 71-79 (2002).

[9] F. Guerra, Broken Replica Symmetry Bounds in the Mean Field Spin Glass Model, Commun. Math. Phys. 233, 1-12 (2003).

[10] M. Talagrand, The Generalized Parisi Formula, Compte Rendu de l'Académie des Sciences, Paris 337, 111-114 (2003).

[11] M. Talagrand, The Parisi formula, Annals of Mathematics, to appear. 
[12] M. Aizenman, R. Sims and S. Starr, Extended variational principle for the Sherrington-Kirkpatrick spin-glass model, Phys. Rev. B68, 214403 (2003).

[13] H.E. Stanley, Introduction to phase transitions and critical phenomena, Oxford University Press, New York and London, 1971.

[14] D. Ruelle, Statistical mechanics. Rigorous results, W.A. Benjamin Inc., New York, 1969.

[15] K. Joag-dev, M.D. Perlman and L.D. Pitt, Association of normal random variables and Slepian's inequality, Annals of Probability 11, 451-455 (1983).

[16] J.-P. Kahane, Une inégalité du type Slepian and Gordon sur les processus gaussiens, Israel J. Math. 55, 109-110 (1986).

[17] G. Parisi, A sequence of approximate solutions to the $S-K$ model for spin glasses, J. Phys. A13, L-115 (1980). 